\title{
Research Paper \\ Inappropriate Medication Use and Related Factors in the Elderly Living in Northern Iran
}

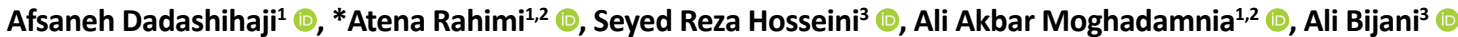

1. Department of Pharmacology and Toxicology, School of Medical, Babol University of Medical Sciences, Babol, Iran.

2. Cellular and Molecular Biology Research Center, Health Research Institute, Babol University of Medical Sciences, Babol, Iran.

3. Social Determinants of Health Research Center, Health Research Institute, Babol University of Medical Sciences, Babol, Iran.

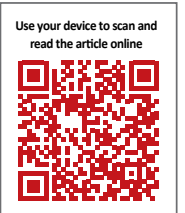

Crtat on: Dadashihaji A, Rahimi A, Hosseini SR, Moghadamnia AK, Bijani A. [Inappropriate Medication Use and Related Factors in the Elderly Living in Northern Iran (Persian)]. Iranian Journal of Ageing. 2021; 15(4):440-457. https://doi.org/10.32598/sija.15.4.1766.1

dol https://doi.org/10.32598/sija.15.4.1766.1

\section{(i) $(8)$}

Received: 15 Jul 2020

Accepted: 29 Nov 2020

Available Online: 24 Feb 2021

Keywords:

Elderly, Drug interactions, Inappropriate medication use, Beers criteria

\section{A B STRACT}

Objectives Older people are more prone to chronic diseases and are being treated than other age groups; as a result, they use more drugs that may be inappropriate and lead to adverse drug reactions. This study aimed to determine the rate of inappropriate drug use using the Beers criterion among the elderly in Amirkola City. Methods \& Materials The current descriptive-analytical study is part of the comprehensive plan "Study of the health status of the elderly in the Amirkola City" AHAP" (No.: 892917) which has been conducted as a Cohort study since 2011 on all people aged 60 and over in the Amirkola City, north of Iran. Necessary information was collected by a trained person using standard questionnaires that included the number, type and duration of drug use. The collected data were analyzed after entering the SPSS statistical software using Chi-square, Fisher's Exact Test, and Logistic Regression to evaluate the status of inappropriate drug. P-value=0.05 was considered as a significant level.

Results The Mean \pm SD age of the elderly was $69.71 \pm 7.47$ years. Prevalence of inappropriate drug use and drugs that should use by caution among the elderly was $37.58 \%$ and $29.85 \%$, respectively. Glibenclamide (12.7\%), Diclofenac (8.8\%) and Clidinium C (5.4\%) were among the most inappropriate drugs used in this population. The highest drug-drug interaction was related to the simultaneous use of two anticholinergic drugs. There was a statistically significant relationship between inappropriate drug use with gender, age, education level, employment status and marriage status $(P<0.05)$.

Conclusion The rate of inappropriate drug use among the elderly in Amirkola is high compared to many other studies that expose the elderly to adverse drug reactions.

\section{Extended Abstract \\ 1. Introduction

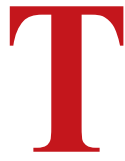 \\ he elderly suffer from chronic diseases more than young adults, which is why \\ multidrug therapy is more common in them. Some of these drugs are considered as inappropriate medications [1]. Nu- merous studies have been conducted around the world and in Iran on the Inappropriate Medication Use (IMU) in the elderly [2-6]. Recent studies have shown that there is a close relationship between the increased IMU and the incidence of physical and cognitive disorders [7, 8]. More studies on the IMU in the elderly can help prevent these complications}

\section{* Corresponding Author:}

Atena Rahimi, PhD.

Address: Department of Pharmacology and Toxicology, School of Medical, Babol University of Medical Sciences, Babol, Iran.

Tel: +98 (11) 32199592

E-mail: atena.rahimi@yahoo.com 
and improve the health status of this population. Since no study has been conducted on the IMU among the elderly in northern Iran, this study aims to investigate the prevalence of IMU in the elderly living in northern Iran and prepare a list of inappropriate medications.

\section{Methods \& Materials}

This study is a part of Amirkala Health and Ageing Project (AHAP) conducted as a cohort study on older adults since 2011. Age $\geq 60$ years, residence in Amirkala county, and willingness to participate in the study were considered as inclusion criteria, while the lack of sufficient information was considered as the exclusion criterion [9]. The required information was collected by a two-part questionnaire with 17 items; the first part consists of 5 questions about the demographic characteristics of participants (age, marital status, level of education, employment status, smoking, underlying diseases, etc.) which was completed through interview, and the second part is a self-report with 12 items assessing the medication information (number and type of medication, and usage duration). Then, the IMU in the elderly was evaluated according to the 2012 Beers criteria [10] regarding the drugs that should not be prescribed in the elderly, the drugs that should be prescribed with caution, drug interaction, and the use of drugs with strong anticholinergic properties. The collected data were statistically analyzed in SPSS version 23 software by using chi-square test, Fisher's exact test, and logistic regression analysis. The $\mathrm{P} \leq 0.05$ was considered as the significance level.

\section{Results}

A total of 777 elderly people aged 60-92 years (Mean age= 69.71 years) participated in this study, of which 437 (56.2\%) were male and $340(43.8 \%)$ were female. A total of 292 older adults (37.58\%) used inappropriate drugs according to Beers criteria. Among the inappropriate drugs, the most used drugs were glibenclamide (12.7\%), diclofenac (8.8\%) and clidinium-C (5.4\%). Table 1 shows the results of chi-

Table 1. Chi-square test results of examining the relationship between demographic variables and the use of inappropriate medications

\begin{tabular}{|c|c|c|c|c|c|}
\hline \multirow{3}{*}{ Variables } & \multirow{3}{*}{ Demographic Factors } & \multicolumn{2}{|c|}{ IMU } & \multirow{3}{*}{ Sig. } & \multirow{3}{*}{ Test Statistic } \\
\hline & & & & & \\
\hline & & No & Yes & & \\
\hline \multirow{3}{*}{ Gender } & Male & $136(31.1)$ & 301 (68.9) & \multirow{3}{*}{$<0.001$} & \multirow{3}{*}{17.76} \\
\hline & & & & & \\
\hline & Female & $156(45.9)$ & $184(54.1)$ & & \\
\hline \multirow{7}{*}{ Age } & $60-64$ & $98(37.5)$ & $163(62.5)$ & \multirow{7}{*}{$<0.001$} & \multirow{7}{*}{6.31} \\
\hline & $65-69$ & $59(36)$ & $105(64)$ & & \\
\hline & $70-74$ & $48(35)$ & $89(65)$ & & \\
\hline & & & & & \\
\hline & $75-79$ & $47(36.4)$ & $82(63.6)$ & & \\
\hline & $80-84$ & $22(40)$ & $33(60)$ & & \\
\hline & $85-99$ & $18(58.1)$ & $13(41.9)$ & & \\
\hline \multirow{3}{*}{ Educational level } & Illiterate & $200(39.4)$ & $308(60.6)$ & \multirow{3}{*}{0.036} & \multirow{3}{*}{3.91} \\
\hline & Elementary and middle school & $71(32.3)$ & $149(67.7)$ & & \\
\hline & High school and university degree & 21 (42.9) & $28(57.1)$ & & \\
\hline \multirow[b]{2}{*}{ Employment status } & Unemployed & $220(75.3)$ & $306(24.7)$ & \multirow[b]{2}{*}{$<0.001$} & \multirow[b]{2}{*}{29.65} \\
\hline & Employed & $72(24.7)$ & $179(75.3)$ & & \\
\hline \multirow{3}{*}{ Marital status } & Married & $243(36.8)$ & $418(63.2)$ & \multirow{3}{*}{0.046} & \multirow{3}{*}{1.26} \\
\hline & & & & & \\
\hline & Not married & $49(42.2)$ & $67(57.8)$ & & \\
\hline
\end{tabular}


Table 2. Logistic regression analysis results for determining the role of factors associated with IMU in the elderly

\begin{tabular}{|c|c|c|c|c|c|}
\hline \multicolumn{2}{|c|}{ Demographic Factors } & \multirow{2}{*}{ Probability } & \multirow{2}{*}{ Odds Ratio } & \multicolumn{2}{|c|}{$95 \% \mathrm{Cl}$} \\
\hline & & & & Lower Bound & Upper Bound \\
\hline \multirow{2}{*}{ Gender } & Male & - & 1 & - & - \\
\hline & Female & 0.043 & 1.427 & 1.011 & 2.015 \\
\hline \multirow{6}{*}{ Age } & $60-64$ & - & 1 & - & - \\
\hline & $65-69$ & 0.457 & 0.845 & 0.543 & 1.316 \\
\hline & $70-74$ & 0.375 & 0.804 & 0.496 & 1.303 \\
\hline & $75-79$ & 0.787 & 0.934 & 0.568 & 1.536 \\
\hline & $80-84$ & 0.973 & 1.012 & 0.516 & 1.985 \\
\hline & $85-99$ & 0.004 & 3.421 & 1.481 & 7.901 \\
\hline \multirow{2}{*}{ Educational level } & Illiterate & - & 1 & - & - \\
\hline & literate & 0.320 & 1.195 & 0.841 & 1.697 \\
\hline \multirow{2}{*}{ Employment status } & Unemployed & - & 1 & - & - \\
\hline & Employed & 0.002 & 0.356 & 0.183 & 0.692 \\
\hline \multirow{2}{*}{ Marital status } & Married & - & 1 & - & - \\
\hline & Not married & 0.248 & 1.319 & 0.824 & 2.112 \\
\hline \multirow{2}{*}{ Multidrug use } & No & - & 1 & - & - \\
\hline & Yes & 0.001 & 4.198 & 2.880 & -6.119 \\
\hline
\end{tabular}

square test for evaluating the relationship between demographic variables and IMU. As can be seen, the amount of used inappropriate drugs was significantly different between older men and women $(\mathrm{P}<0.001)$ where women used more inappropriate drugs than men. The IMU rate was higher in those aged $>80$ years and there was a significant relationship between age and IMU $(\mathrm{P}<0.001)$. Educational level $(\mathrm{P}=0.036)$, employment status $(\mathrm{P}<0.001)$ and marital status $(\mathrm{P}=0.046)$ were another effective factors in IMU. Among the participants in this study, $13(1.7 \%)$ used drugs that interacted with each other according to Beers criteria, where the most drug interaction was related to the concomitant use of anticholinergic drugs. Moreover, it was found that 75 older adults used drugs with anticholinergic properties. The highest frequency was related to Clidinium-C (4.6\%).

Logistic regression analysis was used to determine the role of some variables affecting the IMU in the elderly. The results (Table 2) showed that gender, age $>85$ years, employment status and multidrug use (polypharmacy) had a significant effect on IMU where that the likelihood of using inappropriate drug in women was 1.427 times higher than in men (95\%CI: 1.011-2.015). Subjects over the age of 85 were 3.421 times more likely to use inappropriate drugs than those aged 60-64 years (95\%CI: 1.011-2.015). The likelihood of taking an inappropriate drug in subjects with multidrug use was 4.19 times higher than those with no multidrug use (95\%CI: 2.880-6.119).

\section{Conclusion}

The elderly in northern Iran are relatively exposed to IMU. Age over 85 years, employment status and multidrug use are the predictors of IMU. Older women use inappropriate medications more often than older men. Due to the fact that the side effects of taking inappropriate medications can be serious, proper administration of medications in the elderly is very important. Therefore, it is necessary to provide more educational courses to doctors, nurses and patients, and more supervision by the authorities. 


\section{Ethical Considerations}

Compliance with ethical guidelines

This study was approved by the Ethics Committee of Babol University of Medical Sciences (MUBABOL.HRI. REC.1395.63).

\section{Funding}

This article was extracted from $\mathrm{PhD}$. dissertation of first author at Department of Pharmacology and Toxicology, School of Medical, Babol University of Medical Sciences, Babol.

Authors' contributions

Conceptualization: Seyed Reza Hosseini, Atena Rahimi; Methodology: Afsaneh Dadashi Haji, Ali Akbar Moghaddamnia, Ali Bijani; Investigation: Athena Rahimi, Seyed Reza Hosseini.

\section{Conflicts of interest}

All authors declare no conflict of interest.

\section{Acknowledgements}

The authors would link to thank Vice Chancellor for Research and Technology, Babol University of Medical Sciences. 
This Page Intentionally Left Blank 


\title{
مصرف داروى نامناسب در سالمندان شهر امير كلاو عوامل مرتبط با آن
}

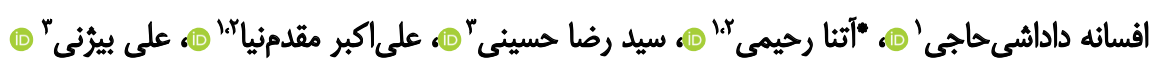

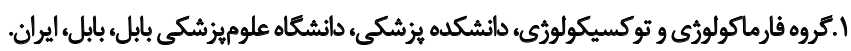

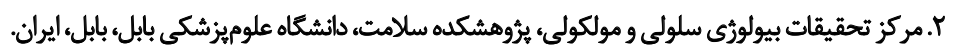

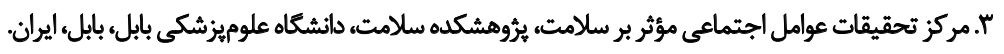

\begin{abstract}
حكبد

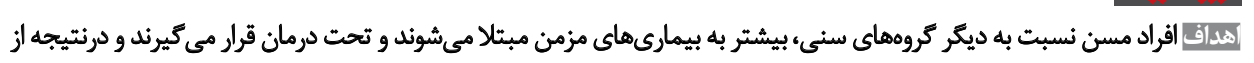

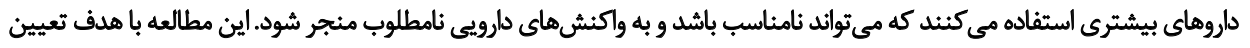

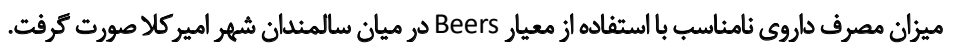

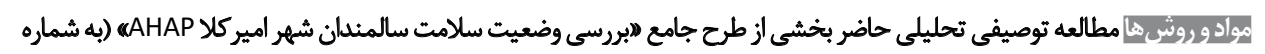

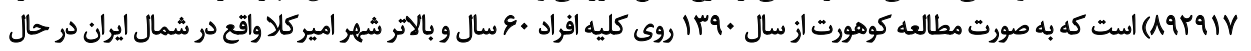

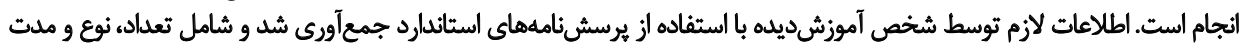

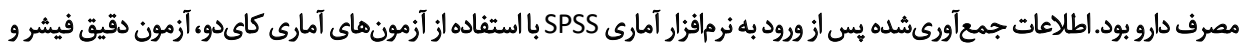

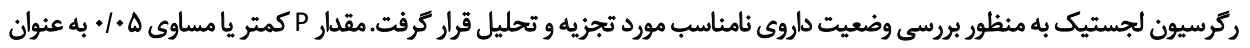
سطح معنادار در نظر كرفته شد.

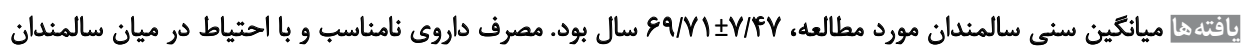

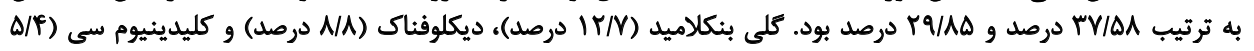

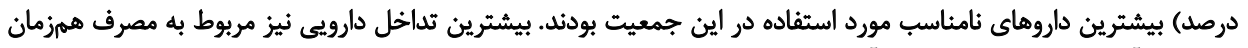

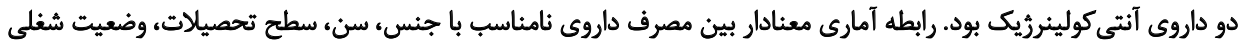

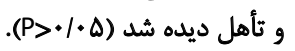

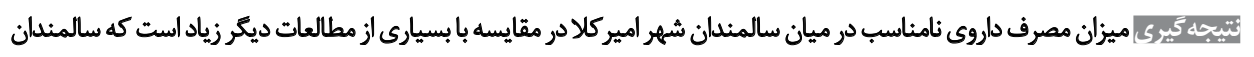

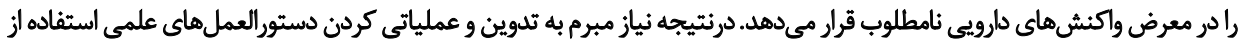

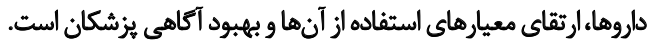

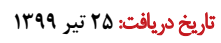

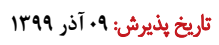
تاريخ انتشار: 1 دى 1499

دارويى، عوارض شديد و تداخلات دارويى نبايد در افراد بالاى

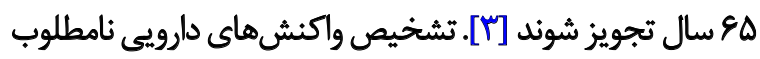

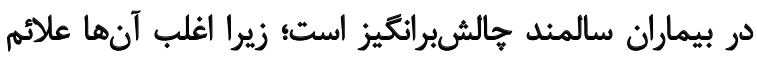

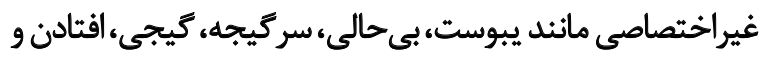

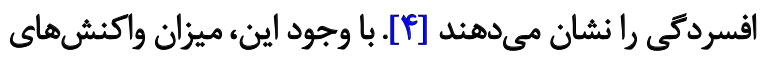

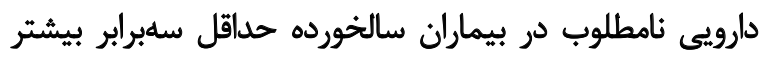

مطالعات جديدى به منظور بررسى ارتباط بين استفاده از

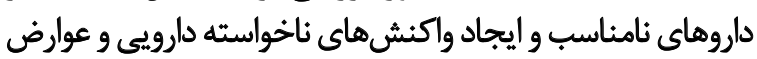

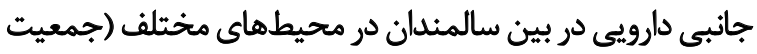
عمومى، سالمندان بسترىشده در بين سالمنار دمريطائ، ساكنين خانه

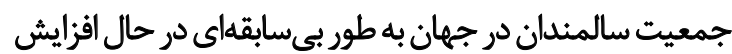
است؛ به طورى كه بيشبيش

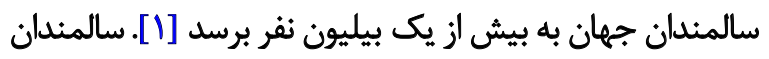

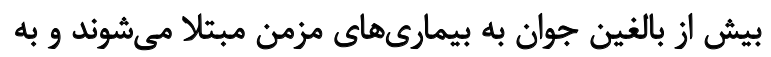

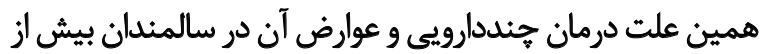

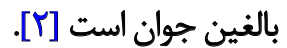
برخى از اين داروها به عنوان داروهاى نامناسب براي سالمندان

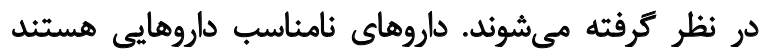
كه به علت اثربخشى يايين و يا ايجاد واكنش نائهاي نامطلوب دارويى، مصرف دارئيدي تاخلي نامناسب، معيار هاي Beers

مقدمه 
افراد سالخورده بيشترى ممكن است در معرض داروهاى نامناسب قرار بكيرند. مطالعات اخير نشان داده كه ارتبن إنباط نزديكيكى ميان

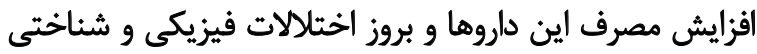

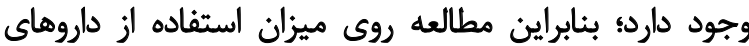

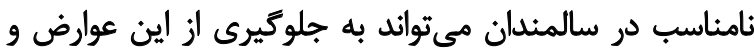
بهبود وضعيث سلامث اين جمعيث كمك كند. از آنجا كه تا كنون تحقيقى در خصوص ميزان مصرف داروى ديان

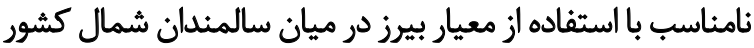

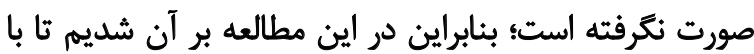

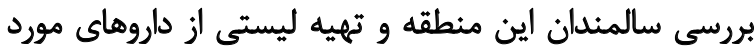

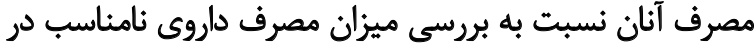
ميان آنان با استفاده از معيار كفتهشده اقدام كنيهم.

$$
\text { روش مطالعه }
$$

اين مقاله به صورت مطالعه كوهورت از سال • وجا روى كليه

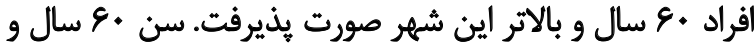

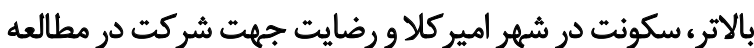

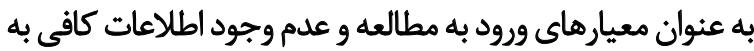

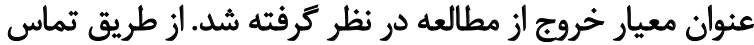

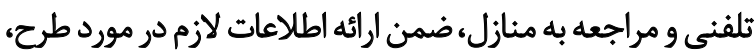

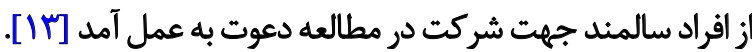
شهر اميركلا دو مركز بهداشتىدرمانى دارد كه ليست كليه

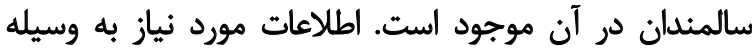

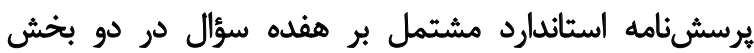

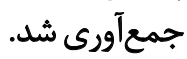

قسمت اول شامل يُنج سؤال در زمينه مشخصات جمعيتشناختى افراد از جمله سن، وضعيت ثأهل، ميزان ديزان

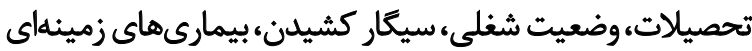

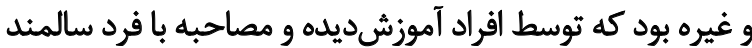
يا در صورت لزوم مصاحبه با نزديكان مطلع وى جمع آورى شيد شدر. قسمت دوم يرسشنامه حاوى دوازده سؤال در رابطه با سابقه

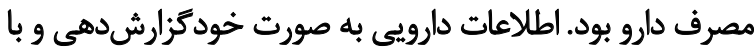

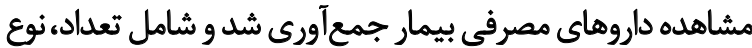

و مدت مصرف دارو بود.

سيّ مصرف داروي نامناسب در سالمندان با توجه به معيار

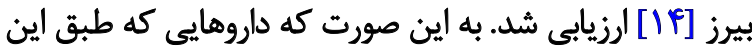

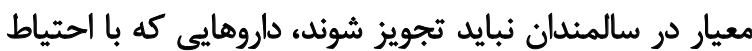

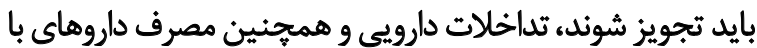

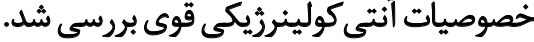

SPSS درنهايت، اطلاعات جمعآورى شده يس از ورود به نرمافزئر

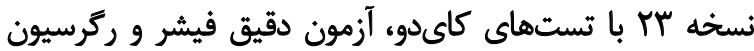

سالمندان) انجام شده است. نتايج اين مطالعات نشان ميدهد

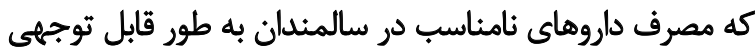
با افزايش واكنشهاى ناخواسته دارويى و عوارض جارئ جانبي داروها

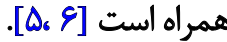

ابزارهاى مختلفى جهت غربالكرى داروهاى نامناسب وجود

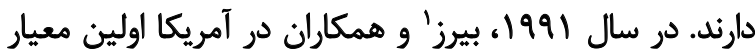

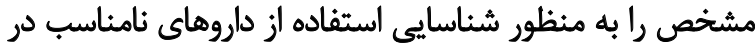

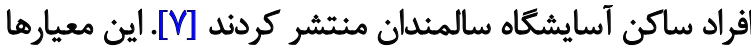

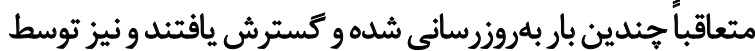

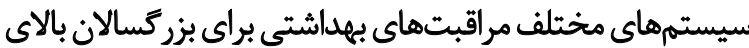

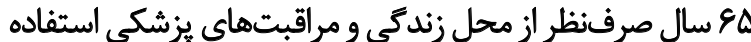

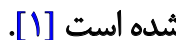

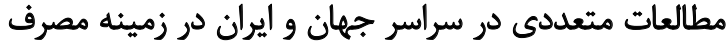

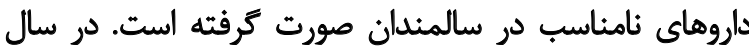

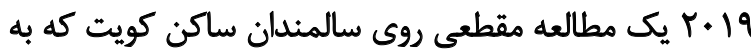

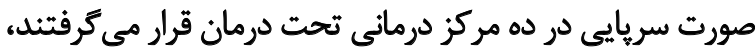

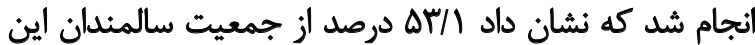

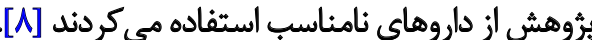

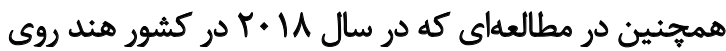

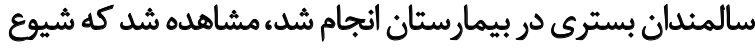

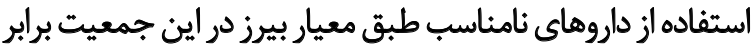

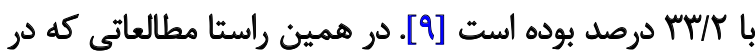

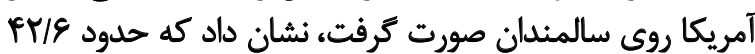

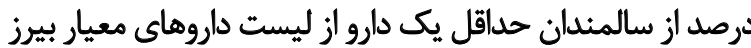

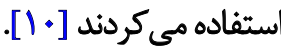
در مطالعاتى كه در ايران صورت كرفت نيز نشان داده شد

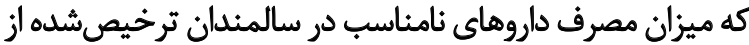

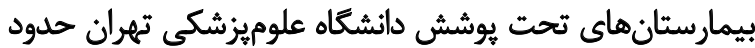

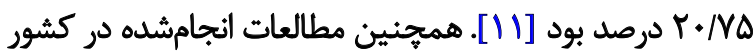

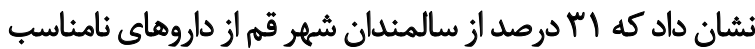

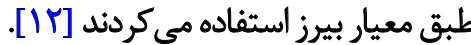
در ايران براى تجويز داروها در سالمندان از معيار بيرز يا

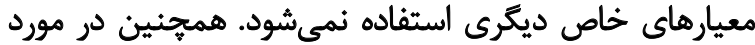

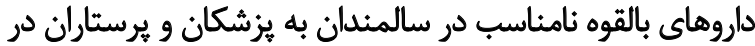

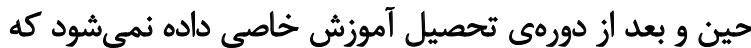

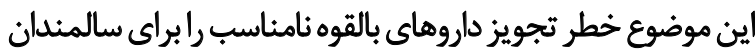
در يى خواهد داشت. از طرف ديكر از آنجا كه جمعيت سالمند در ايران، مشابه روند

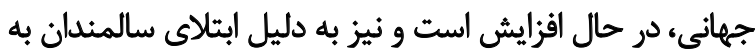

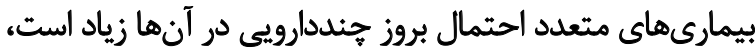




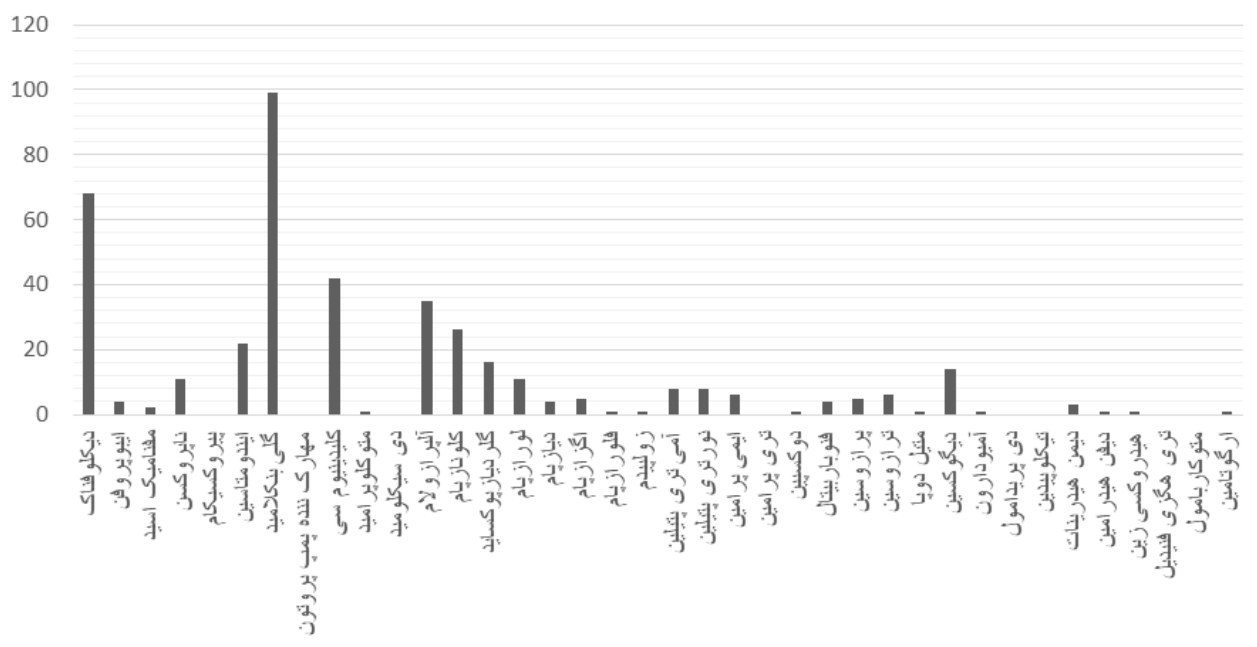

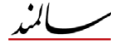

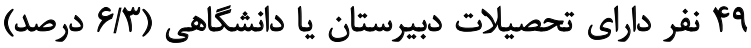

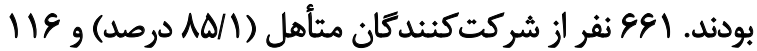

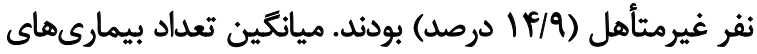

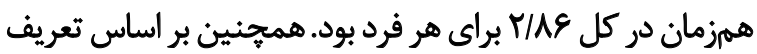

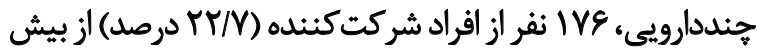

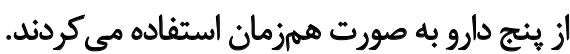

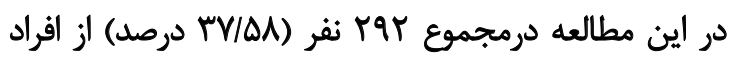

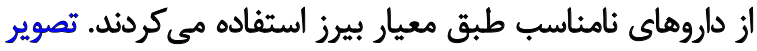

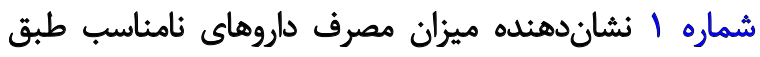

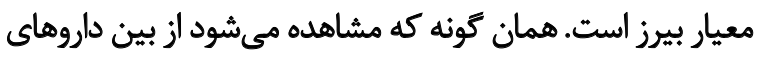

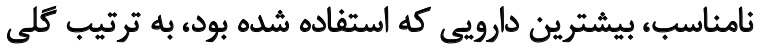

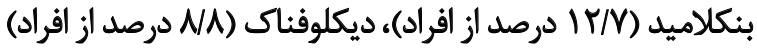
و كليدينيوم سى (

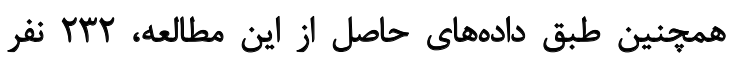

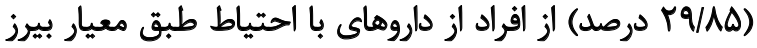

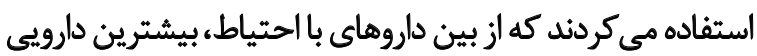

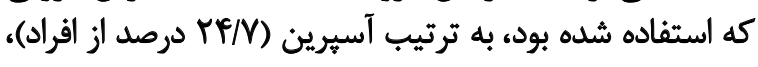

تصوير 1. تعداد افراد استفادهكنده از داروهاى نامناسب طبق معيار Beers لجستيك مورد تجزيه و تحليل آمارى قرار كرفت. مقدار P Pمتر يا مساوى ه ٪ • به عنوان سطح معنادار در نظر كرفته شد.

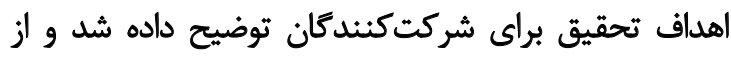

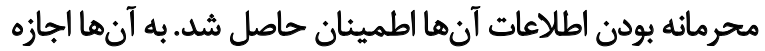
داده شد در هر زمان مطالعه راتهات اطرك كنيند.

يافتهها

در اين مطالعه مجموعاً

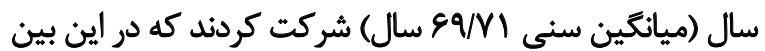

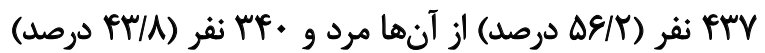

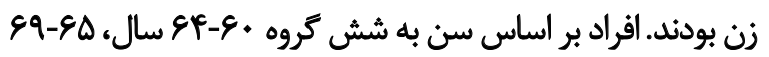

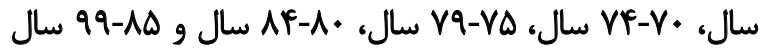

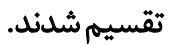

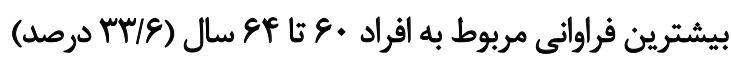

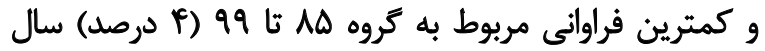

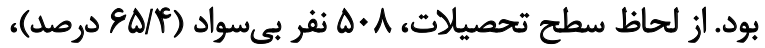

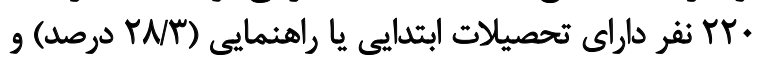

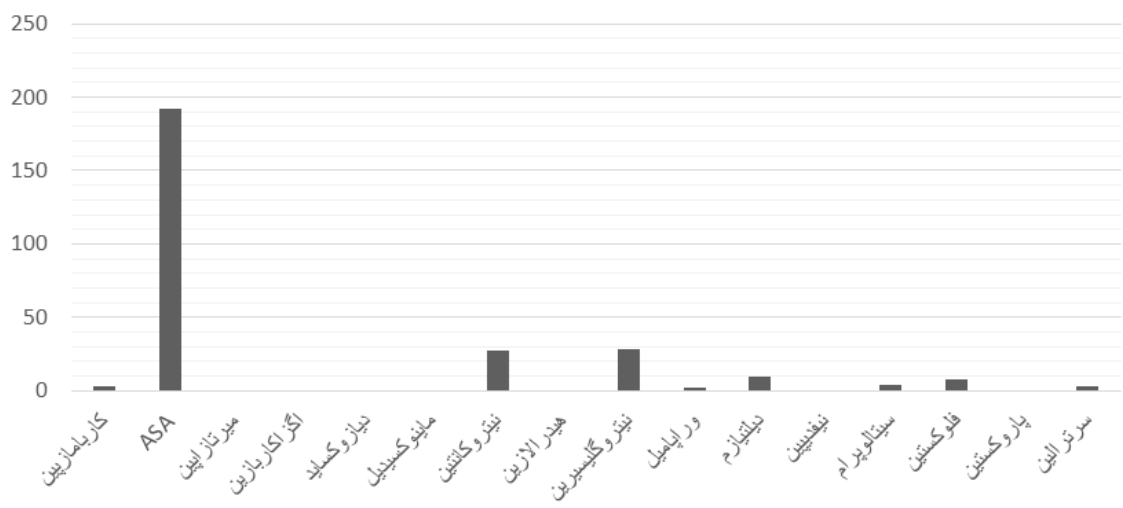


جدول ا. رابطه بين مصرف داروى نامناسب و متغيرهاى جمعيتشناختى در سالمندان مورد مطالعه

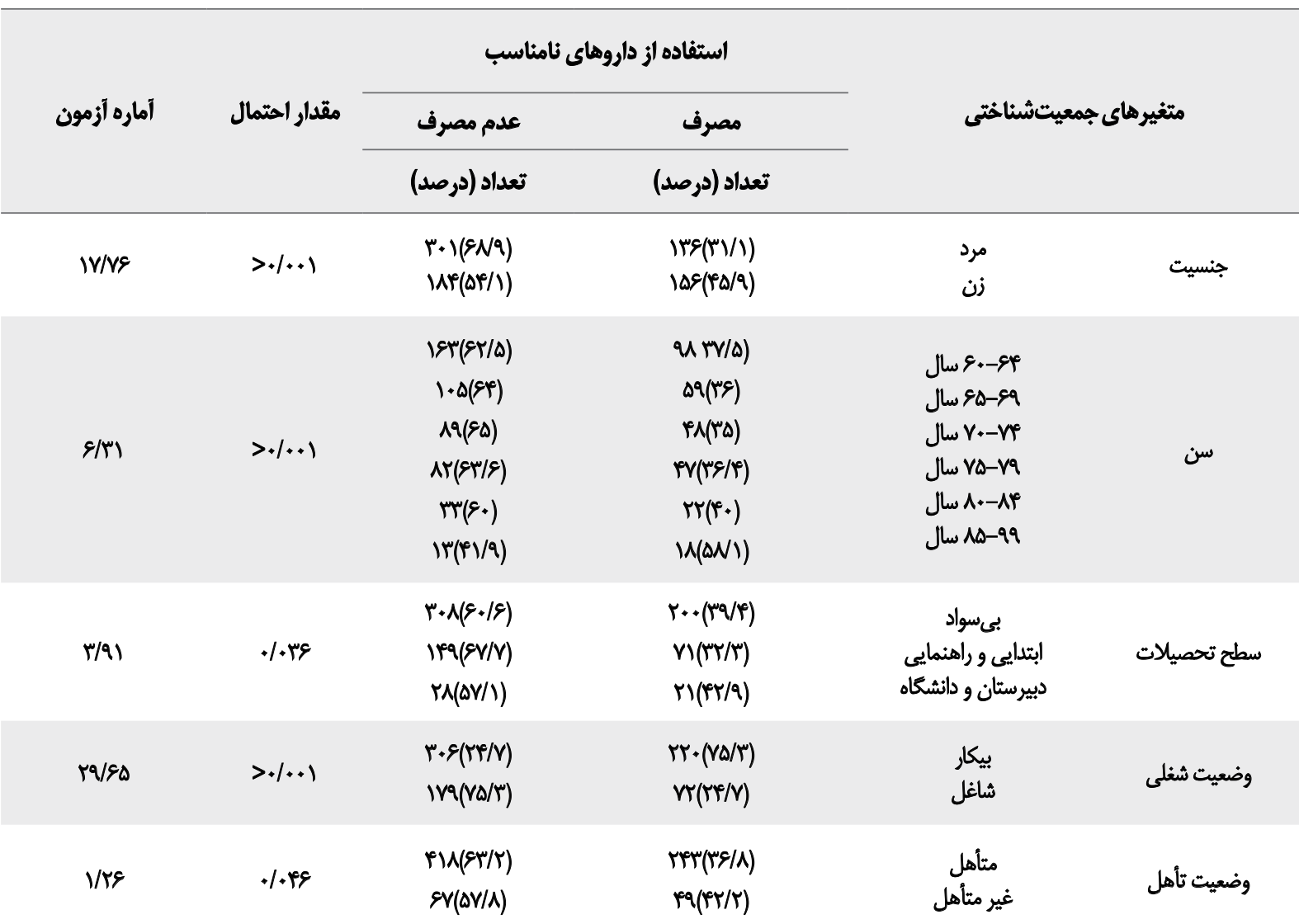

每

(P=/.rr)

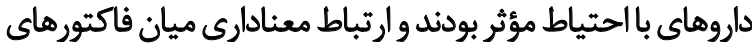

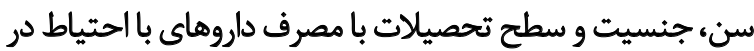
جمعيت مورد مطالعه وجود نداشت.

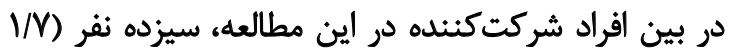

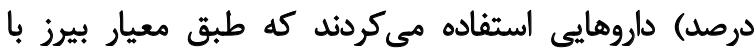

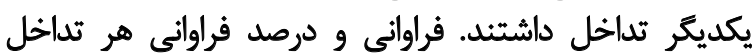

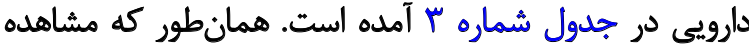

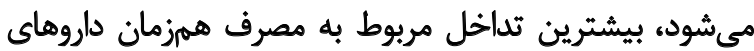
آنتى كولينرزيكاست.

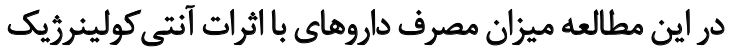

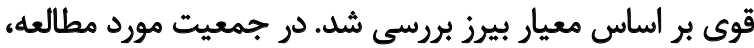

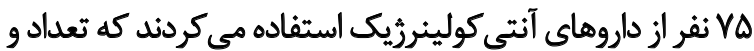

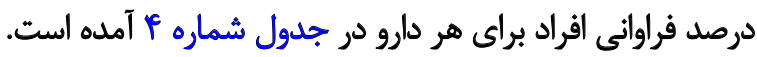

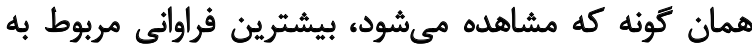

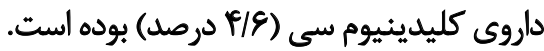
جهت تعيين نقش برخى متغيرهاى تأثير كذار بر مصرف داروى

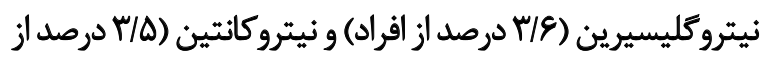

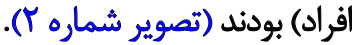

براى بررسى ارتباط متغيرهاى جمعيتشناختى با مصرف

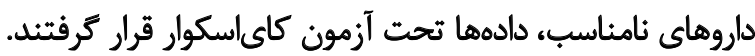
جدول شماره ا نشاندهنده اطلاعات حاصل از ازين آزمان آزمون است

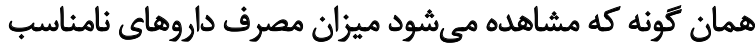

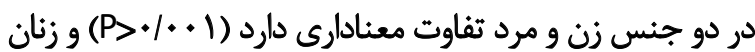
بيشتر از مردان از داروهاى نامناسب استفاده مي كنند.

بر اساس اين نتايج ميزان مصرف داروى نامناسب در سنين

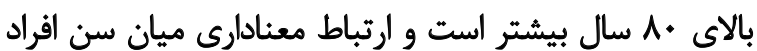

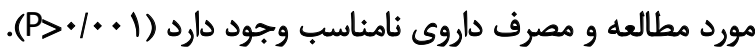

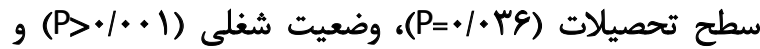

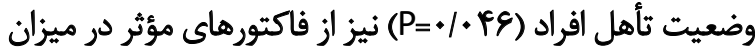
استفاده از داروهاى نامناسب هستند. جدول شماره Y ارتباط ميان متغيرهاى جمعيتشناختى و و و

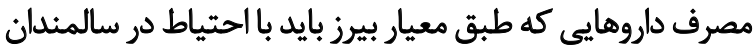

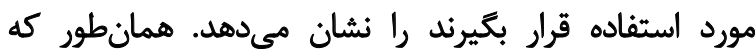

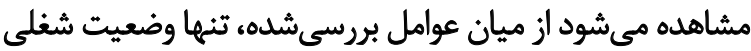


جدول r. رابطه بين مصرف داروى با احتياط و متغيرهاى جمعيتشناحتى در سالمندان مورد مطالعه

\begin{tabular}{|c|c|c|c|c|c|}
\hline \multirow{3}{*}{ أماره آزمون } & \multirow{3}{*}{ مقدار احتمال } & (1) & & \multirow{3}{*}{\multicolumn{2}{|c|}{ متغير هاى جمعيتشُناختى }} \\
\hline & & \multicolumn{2}{|c|}{ استفاده از داروهاى با احثياط } & & \\
\hline & & عدهم مصرف & مصرف & & \\
\hline \multirow{2}{*}{$T / T F$} & \multirow{2}{*}{$.1 . \mathrm{VA}$} & $M / \varepsilon(N / r)$ & $|T|(T V / V)$ & 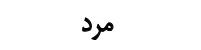 & \multirow{2}{*}{ جنسيت } \\
\hline & & $M r q(E V / P)$ & $111(\pi / 8)$ & زن & \\
\hline \multirow{6}{*}{ NTE } & \multirow{6}{*}{.$/ \Delta Q$} & $194(N / 9)$ & EA (YR/) & fle.-ge & \multirow{6}{*}{ سن } \\
\hline & & $1 . r(E Y / A)$ & el $(M V / Y)$ & ل & \\
\hline & & $q(N \cdot 1)$ & p) (1q/q) & سال V.-V & \\
\hline & & $q(M+/ T)$ & $M(T \Delta / \varphi)$ & wo-Vq & \\
\hline & & $r \Delta(8 T / 8)$ & $r \cdot(r \& / P)$ & (l) A.-Af & \\
\hline & & $M(M / \cdot)$ & $q(r q / \cdot)$ & 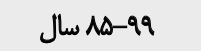 & \\
\hline \multirow{3}{*}{$T / \Delta S$} & \multirow{3}{*}{$.1+11$} & $\operatorname{meg}(N)$ & $\operatorname{Ifr}(\mathrm{TA})$ & بى بسواد & \multirow{3}{*}{ سطح تحصيلات } \\
\hline & & If\& $(E \& / F)$ & $V^{\prime}(\pi+/ 8)$ & ابتدايع و راهنمايى & \\
\hline & & $\Delta f \Delta(N \cdot / 1)$ & $m$ (Tq/) & دييرستان و دانشكاه & \\
\hline \multirow{2}{*}{1.1.} & \multirow{2}{*}{$\%$. H } & $\operatorname{r\Delta V}(\varepsilon \Delta / \Delta)$ & $\operatorname{leq}(N / A)$ & بيكار & \multirow{2}{*}{ وضعيت شغلى } \\
\hline & & $M(M+\Delta)$ & $R(T V / T)$ & شاغل ل ش & \\
\hline \multirow{2}{*}{$1 / \varphi$} & \multirow{2}{*}{ TH. } & $P V \cdot(V / I)$ & $191($ FNQ & مثئهل & \multirow{2}{*}{ وضعيت تأهل } \\
\hline & & $V \Delta(E P / V)$ & $F\left(r \Delta / r^{*}\right)$ & 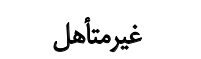 & \\
\hline
\end{tabular}

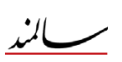

جدول r. تعداد و درصد فراوائى براى استفاده از داروهاى تداخل دار طبق معيار Beers

\begin{tabular}{|c|c|}
\hline تعداد (درصد) & نوع تداخل \\
\hline$r(\cdot / r)$ & كايتويريل تريامترن \\
\hline $1(\cdot / 1)$ & انالايريل ترى امترن \\
\hline$r(\cdot / r)$ & يردينيزولون NSAID \\
\hline$r\left(\cdot / R^{4}\right)$ & أنتى كولينرزيك آنثى كولينيرزيك \\
\hline $1(\cdot / 1)$ & SSRI-NSAID \\
\hline$r(\cdot / r)$ & أنتىسايكوتيك بنزوديازيين \\
\hline $1(\cdot / 1)$ & زولييدم أنتى اييليتيك \\
\hline $1(\cdot / 1)$ & بكلومتازون NSAID \\
\hline
\end{tabular}


جدول F. تعداد و درصد فراوانى داروهاي با اثرات آنتي كولينروُيكى قوى در سالمندان مورد مطالعه

\begin{tabular}{|c|c|}
\hline تعداد (درصد) & نوع داروى آنتى كولينرزيك \\
\hline$q(+/ \Lambda)$ & آمى ترى بتيلين \\
\hline$\lambda(* / 1)$ & نورترى يتيلين \\
\hline$r(\cdot / 4)$ & ايمى بيرامين \\
\hline$r(\cdot / 4)$ & يرفنازين \\
\hline$r(* / 4)$ & ترى هكزئ فنيديل \\
\hline $1(\cdot / 1)$ & دوكسيين \\
\hline$r(\cdot / r)$ & ديمن هيدرينات \\
\hline$r(* / r)$ & هيدروكسى زين \\
\hline $1(* / 1)$ & سييروهيتادين \\
\hline $1(* / 1)$ & ديفن هيدرامين \\
\hline$r q(F / q)$ & كليدينيوم سى \\
\hline $1(* / 1)$ & هيوسين \\
\hline$r(\cdot / r)$ & اكسى بوتينين \\
\hline $1(\cdot / 1)$ & كلرفنيرامين كليدينيوم سى \\
\hline $1(* / 1)$ & ايمى برامين يرفنازين \\
\hline $1(* / 1)$ & آمى ترى يتيلين كليدينيوم سى \\
\hline$r(* / \mu)$ & نورترى يتيلين كليدينيوم سى \\
\hline $1(* / 1)$ & ايمى بيرامين يرفنازين ترى هكزى فنيديل \\
\hline
\end{tabular}

几L

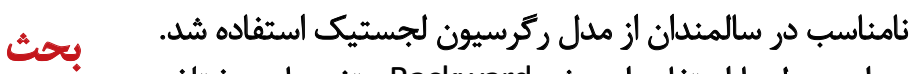

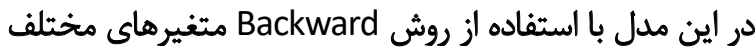
مرتبط با مصرف داروى نامناسب بررسى شدند.

نتايج اين مطالعه نشان داد كه درمجموع YV/هA درصد از

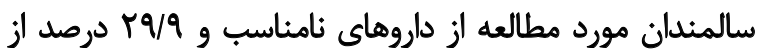

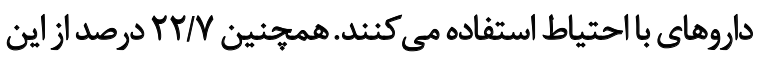

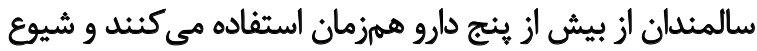

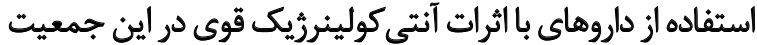

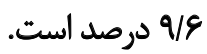

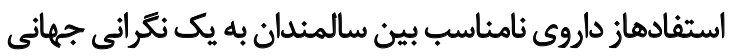

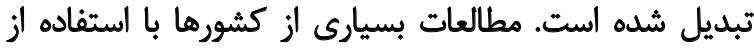

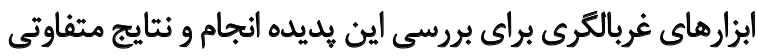

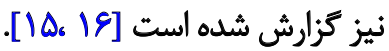

با توجه به يافتههاى اين مطالعه، ميزان مصرف داروهاى

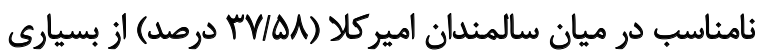

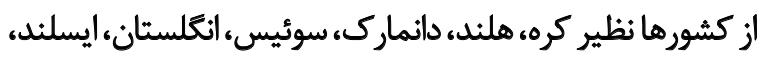

نتايج نشان مي دهد كه متغيرهاي جنس، سن بالاي AD سال،

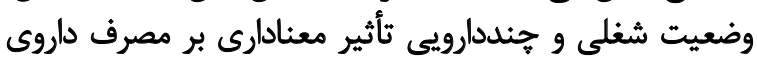

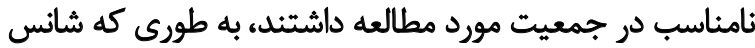

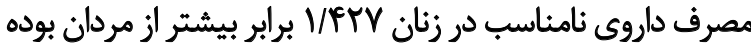

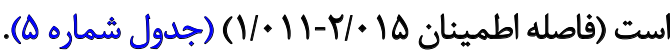

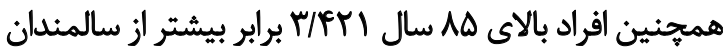

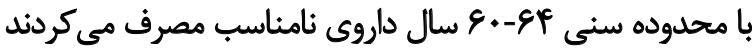

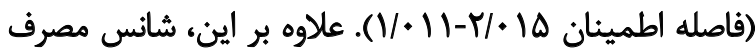

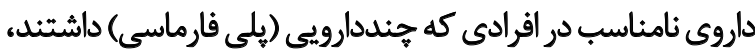

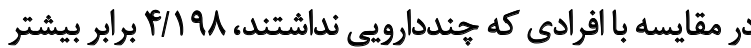
بوده است كه از نظر آمارى معنادار بوده است (فاصله الطمينان

. (T/A1·- - $9 / 119$ 
جدول هـ آناليز ركر سيون لجستيك براى تعيين نقش فاكتورهاى مرتبط با مصرف داروى نامناسب در سالمندان مورد مطالعه

\begin{tabular}{|c|c|c|c|c|c|}
\hline \multicolumn{2}{|c|}{ فاصله اطمينان (ه9 درصد) } & \multirow{2}{*}{ نسبت شانسها } & \multirow{2}{*}{ مقدار الحتمال } & \multirow{2}{*}{\multicolumn{2}{|c|}{ مثغير هاى جمعيتشناختى }} \\
\hline ل حد بالا & حد بايين & & & & \\
\hline- & - & 1 & - & 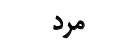 & \\
\hline $5 / \cdot 10$ & $11 \cdot 11$ & V/FT & 1.14 & ن & بلمئ \\
\hline- & - & 1 & - & Jue.-8p & \\
\hline $1 / \pi 18$ & . $/ \Delta F H$ & -/AFD & . FOV & 9 & \\
\hline $1 / r \cdot r$ & - pqe & $\cdot / A \cdot P$ &.$/ M V \Delta$ & (l) $V \cdot-V e$ & \\
\hline V/ArE & - $/ \Delta \& A$ &.$/ 9 m$ & . NAV & سال VD-V9 & 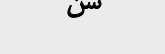 \\
\hline V/UA & .1018 & 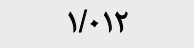 &.$/ 914$ & 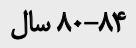 & \\
\hline$V / a+1$ & $|/ F A|$ & M/ATI & $+1++4$ & 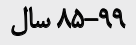 & \\
\hline$=$ & $=$ & 1 & - & بي سواد & 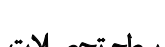 \\
\hline Veav & - $|A P|$ & $1 / 190$ & $\cdot / r T \cdot$ & 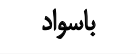 & -2 \\
\hline- & - & 1 & - & 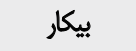 & \\
\hline - leqr & - /MN" & + TAS & $\%$ & 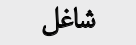 & (2) \\
\hline- & - & 1 & - & متأهل & تات تأهـا \\
\hline tMIt & . AMTF & $1 / 419$ & - MFA & غ غيرمتأهل & \\
\hline- & - & 1 & - & 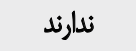 & \\
\hline $8 / 119$ & $r / M$ & $F / 14$ & $+1++1$ & دارند & "جدملرويى \\
\hline
\end{tabular}

L

در مطالعاتى كه در ايران انجام شده بود، بيشترين داروهاى

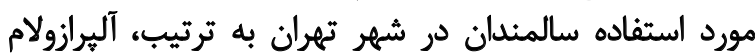

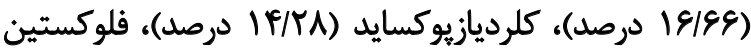

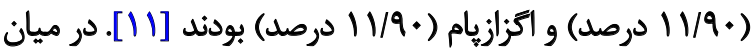

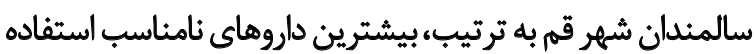

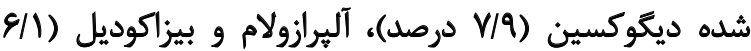

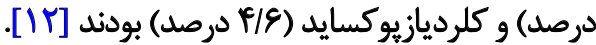
در مطالعه ديكرى در ايران نيز ايندومتاسين، ديفن هيدرامين

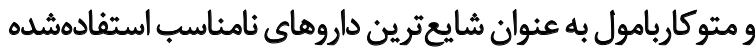

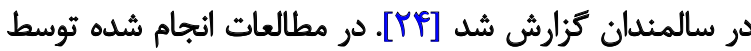

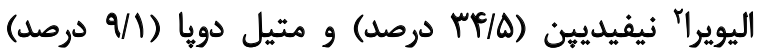

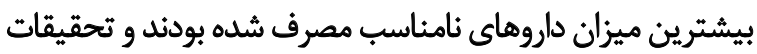

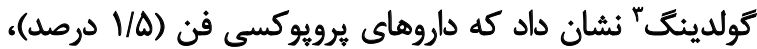

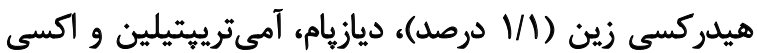

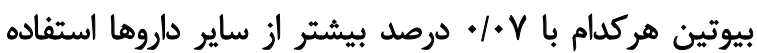

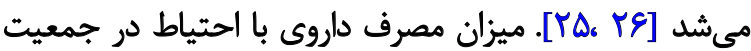

\section{Oliveira}

3. Goulding

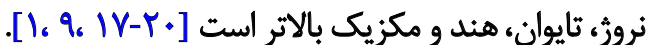

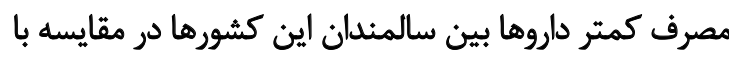

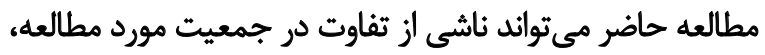

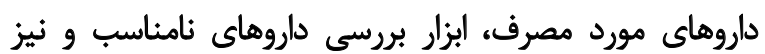

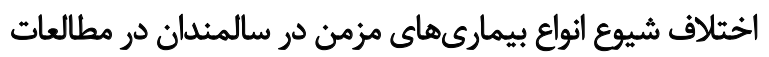

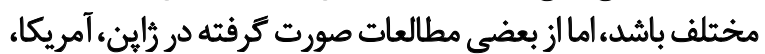

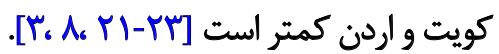

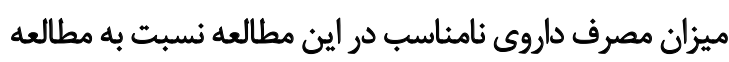

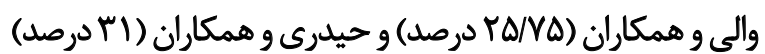

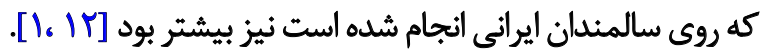

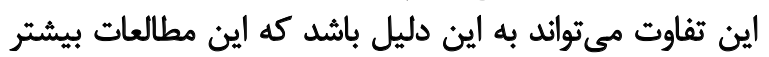

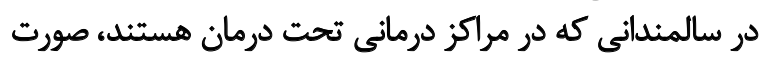

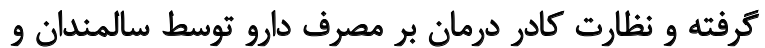

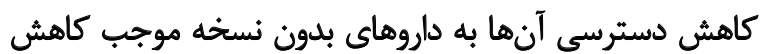
ميزان مصرف داروهاى نامناسب شده است است بلد در مطالعه حاضر از ميان داروهاى نامناسب طبق معيار بيرز

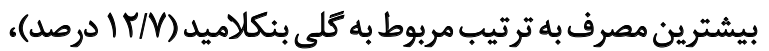

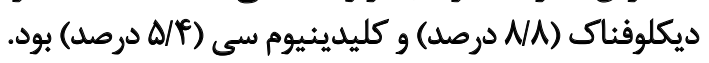




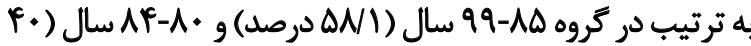

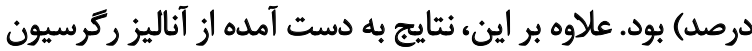

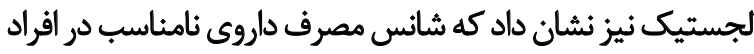

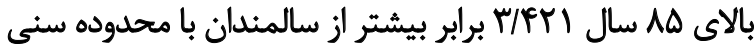

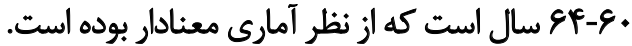

با بالا رفتن سن، تعداد بيمارىهاى همزمان و بالطبع تعداد

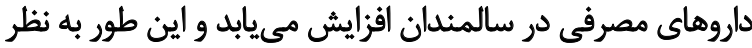

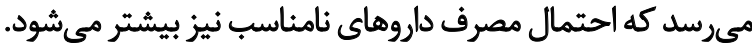

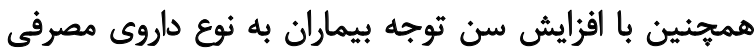

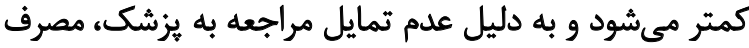

$$
\text { خودسرانه دارو بيشتر مى دشود. }
$$

در مطالعه حاضر TY/ در درصد از سالمندان روزانه بيش از ينج

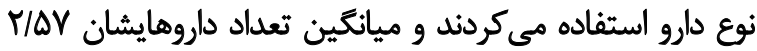

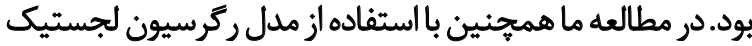

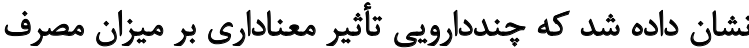
داروهاى نامناسب داشته است.

در مطالعه حيدرى و همكاران، اغلب افراد شركت كنينده در

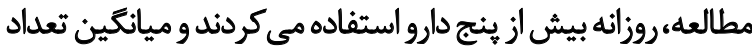

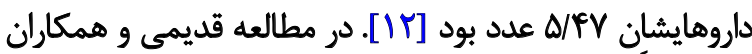

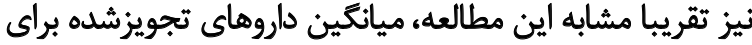

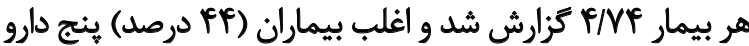

يا بيشتر دريافت مى كردند [TF]

در مطالعات مشابه مطالعه اخير، تعداد داروهاى مصرفي اغلب

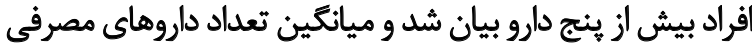

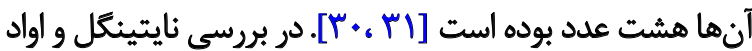

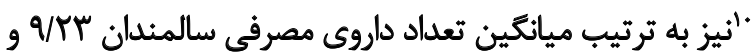

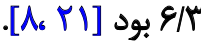

در مطالعه حاضر، بيشترين تعداد داروى بالقوه نامناسب

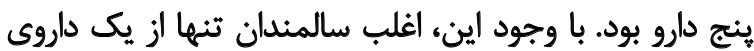

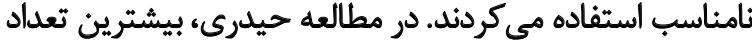

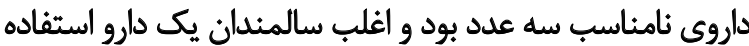

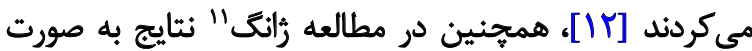

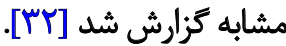

بررسى ها بيرامون ارتباط ميان مصرف داروى نامناسب و وضعيت

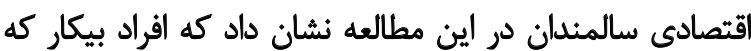

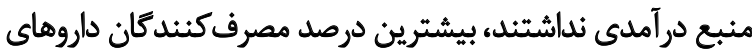

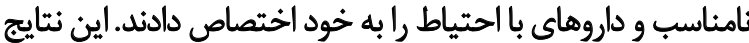

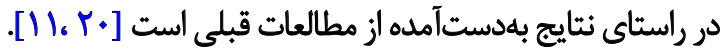
مشكلات اقتصادى نقش مهمى در مصرف نادرست دارو ايفا

10. Nightingale \& Awad

11. Zhang

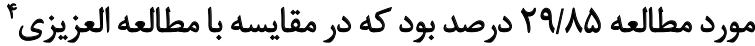

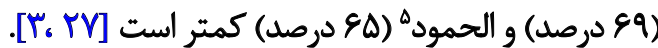

در رابطه با مصرف داروهايى بافعاليت آنتى كولينرزيك در ميان

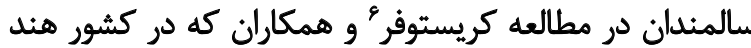

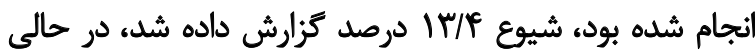

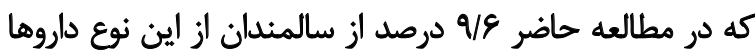

استفاده مى كردند [9].

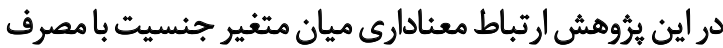

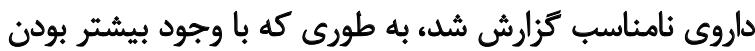

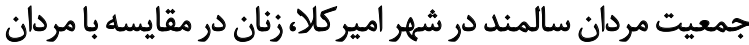
مقادير بالاترى از داروى نامناسب را مصرف ميركير مكردند.

اكرجه در برخى مطالعات مانند مطالعات دهالا و كولدينكَ"

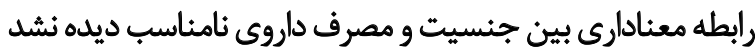

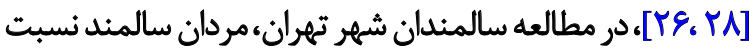

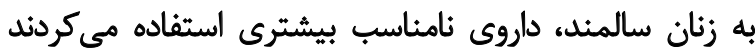

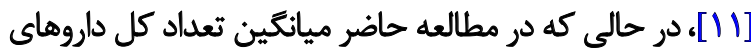

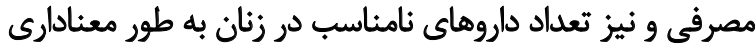

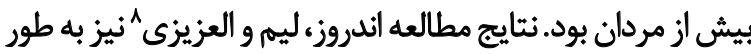

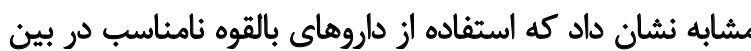

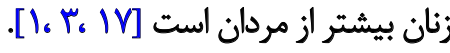

به نظر ميرسد افزايش معنادار تعداد داروها و بيمارىهاي

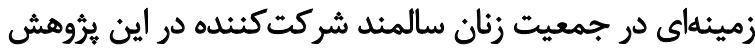

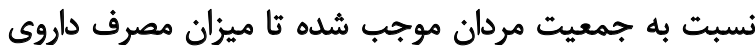

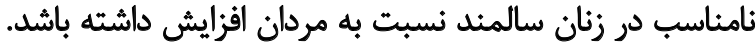

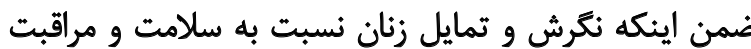

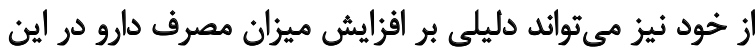

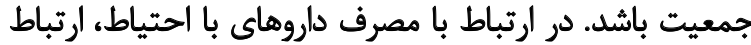
معنادارى با متغير جنسيت مشاهده نشد.

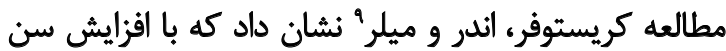

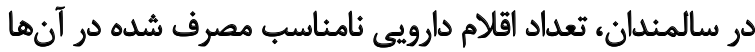

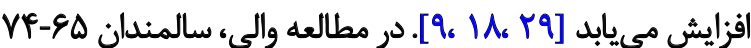

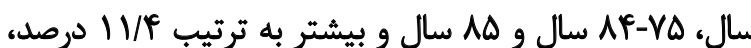

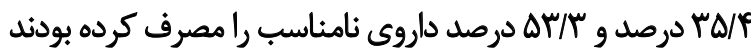

در مطالعه حاضر نيز بين سن بيماران با مصرف داروى نامناسب

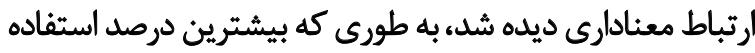
4. Al-Azayzih
5. Alhmoud
6. Christopher
7. Dhalla, Goulding
8. Andrews, Lim \& Al-Azayzih
9. Christopher, Onder \& Miller 
دستورات دارويى و مدت زمان دقيق مصرف داروها به طور دقيق

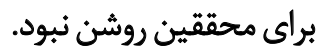

يكى ديكر از محدوديتهاى اين مطالعه عدم وجود دورد اطلاعات

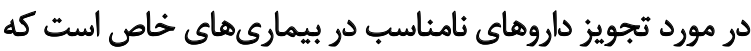

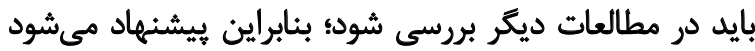

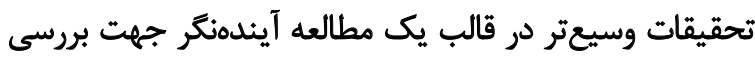
مصرف داروى نامتناسب در سالمندان انجام شود.

\section{نتيجليريرىنهايى}

يافتههاي اين بروهش نشان مى دهد كه جمعيت مورد مطالعه

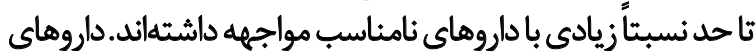

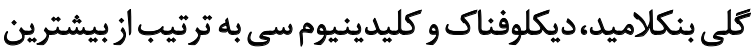

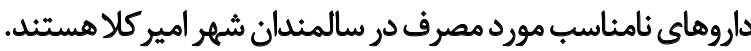

بالاترين ميزان تداخل دارويى هربوط به استفاده از دو داروي

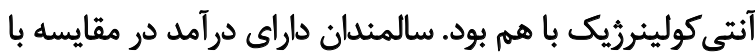

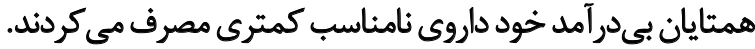

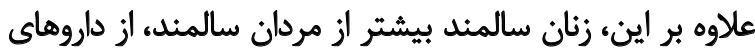

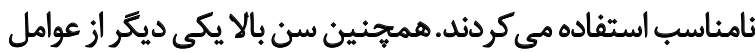
مرتبط با افزايش مصرف داروهاى نامناسب بودين با توجه به اينكه عوارض ناشى از مصرف داروهاى نامناسب

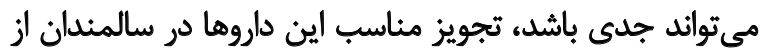

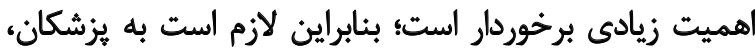

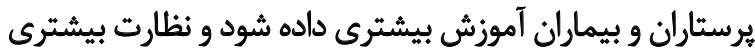
از سوى مسئولين صورت كيرد.

ملاحظات اخلاقى

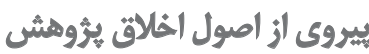

اين مطالعه بخشى از طرح جامع بررسى وضعيت سالمندان

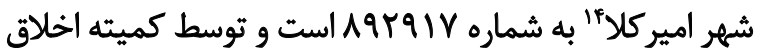

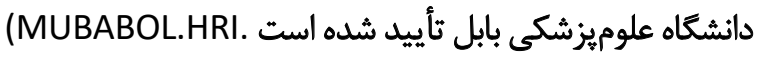
.REC.1395.63)

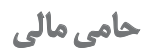

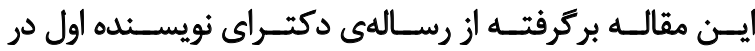

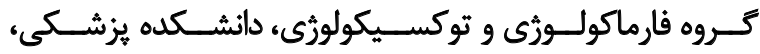

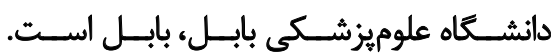

$$
\text { مشاركت نويسند مَّان }
$$

مفهـوم سـازى: سـيد رضـا حسـينى، آتنـا رحيمسي؛ تحقيق

14. Amirkola Health and Ageing Project (AHAP)
مىكند و ميزان مصرف خودسرانه دارو در افراد داراى سطح

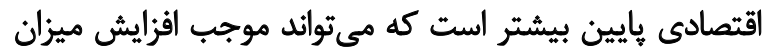

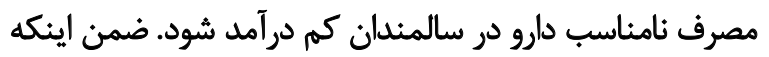

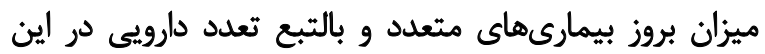
جمعيت از سالمندان نيز بيشتر است.

در اين مطالعه بين سطح تحصيلات و ميزان استفاده از داروى

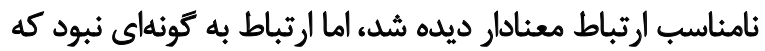

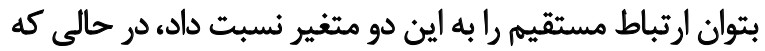

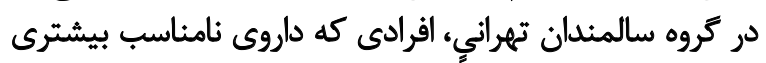

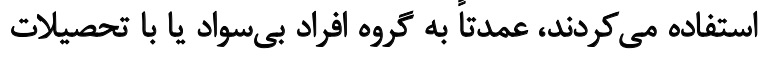

ابتدايى تعلق داشتند [11)]

همجينين در ميان سالمندان شهر قم ديده شد كه مصرف

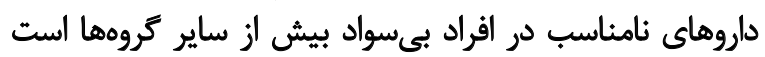

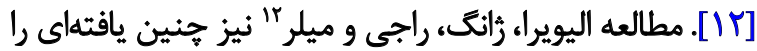

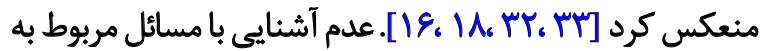

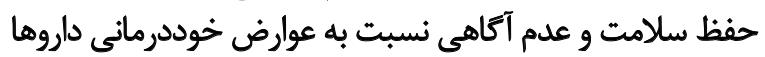

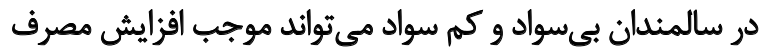
خودسرانه دارو و درنتيجه افزايش ميزان مصرف دئ داروى نامناسب

$$
\text { در اين جمعيت شود. }
$$

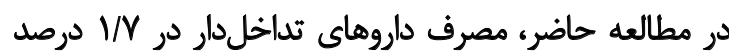

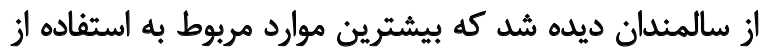

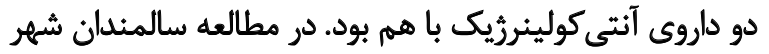

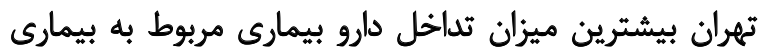

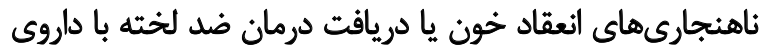

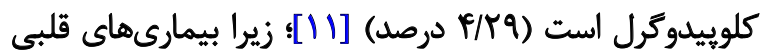

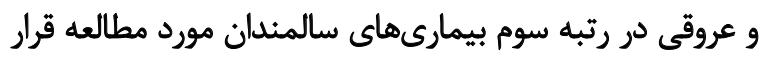
داشتند.

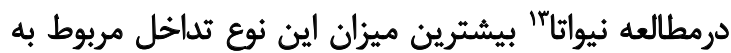

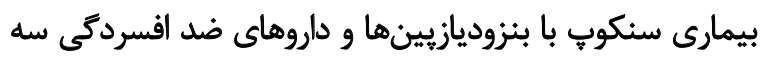

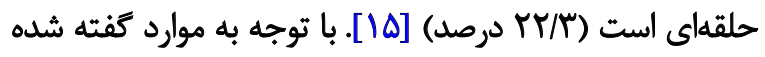

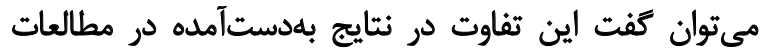

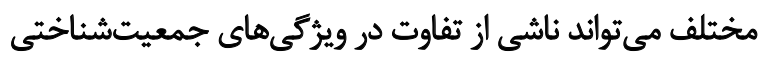

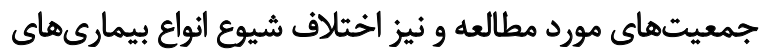

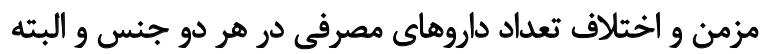

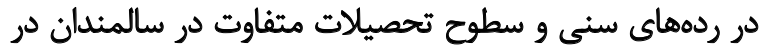
مناطق مختلف جهان باشد.

از نقاط قوت اين مطالعه ميتوان به زياد بودن حجم نمونه

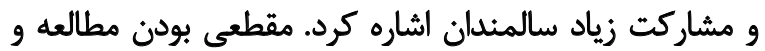

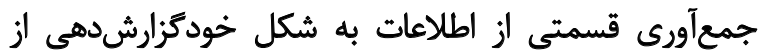

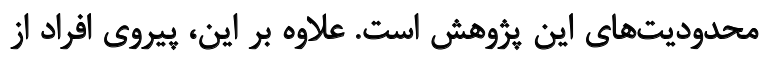

12. Oliveira, Zhang, Raji \& Miller

13. Niwata 


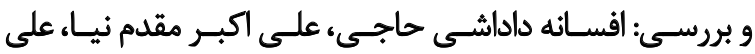

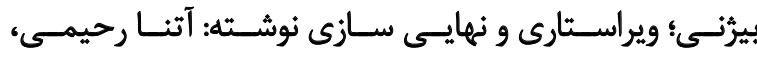

$$
\begin{aligned}
& \text { سـيد رضـا حسينى وين. } \\
& \text { تعارض منافع }
\end{aligned}
$$

بنابر اظهار نويسندكان، اين مقاله تعارض منافع ندارد.

$$
\text { تشكر و قدردانى }
$$

بدينوسيله از معاونت تحقيقات و فناورى دانشكَاه علوم يزشكى تئى بابل جهت حمايت مالى از اين تحقيق و مشاركت سالمندان تصنان تشكر و قدردانى مى كردد. 


\section{References}

[1] Lim YJ, Kim HY, Choi J, Lee JS, Ahn AL, Oh EJ, et al. Potentially inappropriate medications by beers criteria in older outpatients: Prevalence and risk factors. Korean Journal of Family Medicine. 2016; 37(6):329-33. [DOI:10.4082/ kjfm.2016.37.6.329] [PMID] [PMCID]

[2] Nishtala PS, Narayan SW, Wang T, Hilmer SN. Associations of drug burden index with falls, general practitioner visits, and mortality in older people. Pharmacoepidemiology and Drug Safety. 2014; 23(7):753-8. [DOI:10.1002/ pds.3624] [PMID]

[3] Al-Azayzih A, Alamoori R, Altawalbeh SM. Potentially inappropriate medications prescribing according to Beers criteria among elderly outpatients in Jordan: A cross sectional study. Pharmacy Practice (Granada). 2019; 17(2):1439. [DOI:10.18549/PharmPract.2019.2.1439] [PMID] [PMCID]

[4] Grina D, Karpavičiūtè J, Minkutė R, Briedis V. Impact of hospitalization on potentially inappropriate prescribing: A cross-sectional study in an acute geriatric hospital in Lithuania. International Journal of Clinical Pharmacy. 2020; 42(3):903-10. [DOI:10.1007/s11096-020-01035-y] [PMID]

[5] Chang CM, Liu PYY, Yang YHK, Yang YC, Wu CF, Lu FH. Use of the Beers criteria to predict adverse drug reactions among first-visit elderly outpatients. Pharmacotherapy: The Journal of Human Pharmacology and Drug Therapy. 2005; 25(6):831-8. [DOI:10.1592/phco.2005.25.6.831] [PMID]

[6] Perri M, Menon AM, Deshpande AD, Shinde SB, Jiang R, Cooper JW, et al. Adverse outcomes associated with inappropriate drug use in nursing homes. Annals of Pharmacotherapy. 2005; 39(3):405-11. [DOI:10.1345/aph.1E230] [PMID]

[7] Beers MH, Ouslander JG, Rollingher I, Reuben DB, Brooks J, Beck JC. Explicit criteria for determining inappropriate medication use in nursing home residents. Archives of Internal Medicine. 1991; 151(9):1825-32. [DOI:10.1001/ archinte.151.9.1825] [PMID]

[8] Awad A, Hanna O. Potentially inappropriate medication use among geriatric patients in primary care setting: A cross-sectional study using the Beers, STOPP, FORTA and MAI criteria. PloS One. 2019; 14(6):e0218174. [DOI:10.1371/ journal.pone.0218174] [PMID] [PMCID]

[9] Christopher A, Pranita V, Mathews KP, Surekha V, Gopinath K, Gowri M. Potentially inappropriate medication use in the elderly in a tertiary care centre in South India. Journal of the Indian Academy of Geriatrics. 2018; 14(3):130-5. [DOI:10.35262/jiag.v14i3.130-135]

[10] Davidoff AJ, Miller GE, Sarpong EM, Yang E, Brandt N, Fick DM. Prevalence of potentially inappropriate medication use in older adults using the 2012 Beers criteria. Journal of the American Geriatrics Society. 2015; 63(3):486-500. [DOI:10.1111/jgs.13320] [PMID] [PMCID]

[11] Vali L, Pour Reza A, Rahimi Foroushani A, Ahmadi B, Akbari Kamrani AA. [Analysis of inappropriate medication use in older adults discharged from hospitals affiliated with Tehran University of Medical Sciences (TUMS) using the Beers criteria in 2010 (Persian)]. Salmand: Iranian Journal of Ageing. 2011; 6(3):56-65. http:/ / salmandj.uswr.ac.ir/article1-438-en.html
[12] Heidari S, Aliabadi A, Naebi A, Khoramirad A. [Frequency of use of potentially inappropriate medications and its associated factors in elderly people in Qom city, 2012, Iran (Persian)]. Qom University of Medical Sciences Journal. 2014; 8(4):44-52. http://journal.muq.ac.ir/article-1-407-en.html

[13] Hosseini SR, Bayani MA, Mohammadi K, Mohammadi E, Bijani A. [Correlation between anthropometric indexes and risk factors of cardiovascular diseases among the elderly population in Amirkola (Persian)]. Feyz Journal of Kashan University of Medical Sciences. 2017; 21(3):272-9. http:/ / feyz.kaums.ac.ir/article1-3057-en.html

[14] Campanelli CM. American Geriatrics Society updated beers criteria for potentially inappropriate medication use in older adults: The American geriatrics society 2012 Beers criteria update expert ppanel. Journal of the American Geriatrics Society. 2012; 60(4):616-31. [DOI:10.1111/j.1532-5415.2012.03923.x] [PMID] [PMCID]

[15] Niwata S, Yamada Y, Ikegami N. Prevalence of inappropriate medication using Beers criteria in Japanese long-term care facilities. BMC Geriatrics. 2006; 6:1. [DOI:10.1186/1471-2318-6-1] [PMID] [PMCID]

[16] Raji MA, Ostir GV, Markides KS, Espino DV, Goodwin JS. Potentially inappropriate medication use by elderly Mexican Americans. Annals of Pharmacotherapy. 2003; 37(9):1197-202. [DOI:10.1345/aph.1C480] [PMID]

[17] Andrews H, Betz M, Chihuri S, DiGuiseppi C, Eby D, Gordon $\mathrm{A}$, et al. Prevalence of potentially inappropriate medication use in older drivers: AAA longROAD study. 2018. https://trid.trb. org/view/1570170

[18] Miller GE, Sarpong EM, Davidoff AJ, Yang EY, Brandt NJ, Fick DM. Determinants of potentially inappropriate medication use among community-dwelling older adults. Health Services Research. 2017; 52(4):1534-49. [DOI:10.1111/1475-6773.12562] [PMID] [PMCID]

[19] Blozik E, Rapold R, von Overbeck J, Reich O. Polypharmacy and potentially inappropriate medication in the adult, community-dwelling population in Switzerland. Drugs \& Aging. 2013; 30(7):561-8. [DOI:10.1007/s40266-013-0073-0] [PMID]

[20] Fialová D, Topinková E, Gambassi G, Finne-Soveri H, Jónsson PV, Carpenter I, et al. Potentially inappropriate medication use among elderly home care patients in Europe. JAMA. 2005; 293(11):1348-58. [DOI:10.1001/jama.293.11.1348] [PMID]

[21] Nightingale G, Hajjar E, Swartz K, Andrel-Sendecki J, Chapman A. Evaluation of a pharmacist-led medication assessment used to identify prevalence of and associations with polypharmacy and potentially inappropriate medication use among ambulatory senior adults with cancer. Journal of Clinical Oncology. 2015; 33(13):1453-9. [DOI:10.1200/JCO.2014.58.7550] [PMID]

[22] Wessell AM, Nietert PJ, Jenkins RG, Nemeth LS, Ornstein SM. Inappropriate medication use in the elderly: Results from a quality improvement project in 99 primary care practices. The American Journal of Geriatric Pharmacotherapy. 2008; 6(1):21-7. [DOI:10.1016/j.amjopharm.2008.02.001] [PMID]

[23] Lai H-Y, Hwang S-J, Chen Y-C, Chen T-J, Lin M-H, Chen L-K. Prevalence of the prescribing of potentially inappropriate medications at ambulatory care visits by elderly patients covered by the Taiwanese National Health Insurance program. Clinical Therapeutics. 2009; 31(8):1859-70. [DOI:10.1016/j.clinthera.2009.08.023] [PMID] 
[24] Ghadimi H, Esmaily HM, Wahlstrom R. General practitioners' prescribing patterns for the elderly in a province of Iran. Pharmacoepidemiology and Drug Safety. 2011; 20(5):482-7. [DOI:10.1002/pds.2106] [PMID]

[25] de Oliveira Baldoni A, Ayres LR, Martinez EZ, Dewulf NdLS, dos Santos V, Pereira LRL. Factors associated with potentially inappropriate medications use by the elderly according to Beers criteria 2003 and 2012. International Journal of Clinical Pharmacy. 2014; 36(2):316-24. [DOI:10.1007/s11096-013-9880-y] [PMID]

[26] Goulding MR. Inappropriate medication prescribing for elderly ambulatory care patients. Archives of Internal Medicine. 2004; 164(3):305-12. [DOI:10.1001/archinte.164.3.305] [PMID]

[27] Alhmoud E, Khalifa S, Bahi AA. Prevalence and predictors of potentially inappropriate medications among home care elderly patients in Qatar. International Journal of Clinical Pharmacy. 2015; 37(5):815-21. [DOI:10.1007/s11096-015-0125-0] [PMID]

[28] Dhalla IA, Anderson GM, Mamdani MM, Bronskill SE, Sykora $\mathrm{K}$, Rochon PA. Inappropriate prescribing before and after nursing home admission. Journal of the American Geriatrics Society. 2002; 50(6):995-1000. [DOI:10.1046/j.1532-5415.2002.50252.x] [PMID]

[29] Onder G, Landi F, Cesari M, Gambassi G, Carbonin P, Bernabei R. Inappropriate medication use among hospitalized older adults in Italy: Results from the Italian group of pharmacoepidemiology in the elderly. European Journal of Clinical Pharmacology. 2003; 59(2):157-62. [DOI:10.1007/s00228-003-0600-8] [PMID]

[30] Cannon KT, Choi MM, Zuniga MA. Potentially inappropriate medication use in elderly patients receiving home health care: A retrospective data analysis. The American Journal of Geriatric Pharmacotherapy. 2006; 4(2):134-43. [DOI:10.1016/j.amjopharm.2006.06.010] [PMID]

[31] Vanier A, Paille C, Abbey H, Berrut G, Lombrail P, Moret L. Assessment of inappropriate prescribing in the elderly subject during acute care hospitalisation. Gériatrie et Psychologie Neuropsychiatrie du Vieillissement. 2011; 9(1):51-7. [DOI:10.1684/ pnv.2011.0256] [PMID]

[32] Zhang YJ, Liu WW, Wang JB, Guo JJ. Potentially inappropriate medication use among older adults in the USA in 2007. Age and Ageing. 2011; 40(3):398-401. [DOI:10.1093/ageing/afr012] [PMID]

[33] Oliveira MG, Amorim WW, de Jesus SR, Rodrigues VA, Passos LC. Factors associated with potentially inappropriate medication use by the elderly in the Brazilian primary care setting. International Journal of Clinical Pharmacy. 2012; 34(4):626-32. [DOI:10.1007/s11096-012-9656-9] [PMID] 
This Page Intentionally Left Blank 